LA GRANJA:

REVISTA DE

CIENCIAS DE LA VIDA

pISSN:1390-3799; eISSN:1390-8596

http:/ / doi.org/10.17163/lgr.n27.2018.06
Artículo científico / Scientific paper

ECOLOGÍA Forestal

\title{
POBLACIÓN DE MACROFAUNA EN SISTEMAS SILVOPASTORILES DEDICADOS A LA PRODUCCIÓN LECHERA:ANÁLISIS PRELIMINAR
}

\author{
MACROFAUNA POPULATION IN SILVOPASTORAL SYSTEMS FOR MILK \\ PRODUCTION: PRELIMINARY ANALYSIS
Diego Marcelo Caicedo Rosero ${ }^{1}$, Hernán Rigoberto Benavides Rosales ${ }^{1}$, Luis Alfredo Carvajal Pérez ${ }^{1}$, Jessica Paola Ortega Hernández ${ }^{1}$

${ }^{1}$ Universidad Politécnica Estatal del Carchi, Telf. 062224 079, Calle Antisana y Av. Universitaria, Tulcán, Ecuador.

*Autor para correspondencia: diego.caicedo@upec.edu.ec

\begin{abstract}
Resumen
En esta investigación se presenta un estudio preliminar de la macrofauna en suelos silvopastoriles dedicados a la producción de leche, cuyo objetivo es analizar las poblaciones de biota del suelo en sistemas silvopastoriles con las siguientes combinaciones: aliso (Alnus acuminata) y mezcla forrajera, acacia (Acacia melanoxylon) y mezcla forrajera, y un testigo solo con mezcla forrajera. En una primera etapa se realizaron 30 muestras en cada población muestral, en los meses de baja precipitación, cada muestra es un cuadrante de $30 \mathrm{~cm} \times 30 \mathrm{~cm} \times 30 \mathrm{~cm}$, en el cual se contabilizó la biota existente. Los datos de las poblaciones se analizaron usando los índices de biodiversidad como riqueza de Margalef, índice de dominancia de Berger-Parker, el índice de dominancia de Simpson y el índice de biodiversidad de Shannon, datos que permitieron concluir que la variación en número de especies no es significativa entre poblaciones muestrales y la existencia de biota mantiene la productividad. Además, estos datos servirán en una segunda investigación para hacer comparaciones estadísticas del número de especies en meses de mayor y menor precipitación.

Palabras claves: Biota, comparación, índices de biodiversidad, Carchi, Ecuador.
\end{abstract}

LA GRANJA: Revista de Ciencias de la Vida 27(1) 2018:77-85.

(C)2018, Universidad Politécnica Salesiana, Ecuador. 


\begin{abstract}
This research shows a preliminary study of macrofauna in silvopastoral soils for milk production, whose objective was to evaluate the soil's population biota in silvopastoral systems whit next combinations: aliso (Alnus acuminata) and fodder mixture, acacia (Acacia melanoxylon) and fodder mixture, and control only fodder mixture. In first stage, 30 samples were collected in each population sample, in months with less precipitation, each sample, is one square of $30 \mathrm{~cm} \times 30 \mathrm{~cm} \times 30 \mathrm{~cm}$, in which counted the existing biota. Population data was analyzed using biodiversity index like Margalef index of species richness, Berger Parker index of dominance, Simpson's dominance index and Shannon diversity index, data which permitted conclude the variation in species number is not significant in population samples and existing biota maintains the productivity. Also, those values will use in a second research to make statistical comparisons in the number of species in months with more and less precipitation.
\end{abstract}

Keywords: Biota, biodiversity index, comparison, Carchi, Ecuador.

Forma sugerida de citar: Caicedo Rosero, D. M., Benavides Rosales, H. R., Carvajal Pérez, L. A., Ortega Hernández, J. P. 2018. Población de macrofauna en sistemas silvopastoriles dedicados a la producción lechera:análisis preliminar. La Granja: Revista de Ciencias de la Vida. Vol. 27(1):77-85. http://doi.org/10.17163/lgr.n27.2018.06. 


\section{Introducción}

De acuerdo con la investigación preliminar (Hernández Sampieri, Fernández Collado y Baptista Lucio, 2014) realizada a los muestreos en los sistemas silvopastoriles de la Finca "San Vicente", parroquia "El Carmelo" provincia del Carchi, se deduce que la productividad de los sistemas silvopastoriles depende de las condiciones climáticas, edáficas y de la presencia de fauna de suelo, esto a su vez influye en la productividad del hato lechero.

En esta investigación se realiza un análisis específico sobre la biota del suelo en función de índices de biodiversidad alfa (Lazo et al., 2007); el objetivo es medir los niveles de producción en los sistemas silvopastoriles, comparando la eficiencia simbiótica de fauna edáfica en pastos con alisos, pastos con acacias y un testigo solo de pastos.

Tal como manifiesta Jordán López (2005-2006) la biota del suelo es importante, ya que de su presencia depende la fertilidad y estabilidad del mismo, lleva a cabo la fragmentación de residuos orgánicos, permite la degradación de los compuestos orgánicos a moléculas más sencillas, actúa sobre la porosidad del suelo, facilita la agregación de partículas del suelo a través de las excreciones e interviene en la humificación de la materia orgánica.

Los análisis realizados en el sitio de estudio sirvieron para identificar la variación en el número poblacional de las especies entre los sistemas silvopastoriles de acuerdo a las mejores relaciones simbióticas entre pastos y árboles.

En este tipo de estudio los índices de biodiversidad se constituyen en herramientas para analizar el equilibrio ecológico entre las especies presentes en los ecosistemas, lo que permite a su vez, demostrar la variación en la calidad de los hábitats (Thevathasan et al., 2014).

El número de especies es la medida más utilizada para los análisis de biodiversidad, porque la riqueza de especies refleja aspectos de la biota, las especies son de fácil identificación y contabilización, y, aunque no se tenga un conocimiento taxonómico completo (especialmente para taxones como, insectos y otros invertebrados) es suficiente tener la disponibilidad bibliográfica sobre especies (Moreno, 2001).

\section{Materiales y métodos}

En base a la información tomada del Sistema de Posicionamiento Global (GPS), la zona de estudio se encuentra en la Finca "San Vicente", parroquia "El Carmelo" provincia del Carchi, localizada en las coordenadas UTM: Lat N 210000, y Long. 10072464. El clima es ecuatorial de alta montaña, con una precipitación entre 1200 y $2000 \mathrm{~mm}$, con un rango de temperatura de $10{ }^{\circ} \mathrm{C}$ a $12^{\circ} \mathrm{C}$ (Estación meteorológica El Carmelo). Conforme a los análisis de suelos, éstos pertenecen al orden inceptisoles con alto contenido de materia orgánica, van de profundos a superficiales bien drenados, buena retención de humedad, textura franco-arenosa, color en húmedo negro, raíces abundantes por la presencia de pastos, pH 6.5, material parental proveniente de cenizas y arenas volcánicas, encontrados en un relieve ondulado y con pendientes que varían entre un $20 \%$ a $25 \%$.

El objetivo de esta investigación es un análisis descriptivo que permita comparar la abundancia de fauna edáfica en sistemas silvopastoriles, este análisis se lo debe hacer dos veces por año, para observar el proceso evolutivo de la biota del suelo. La primera colecta de muestras se realizó en el mes de julio 2016, cuyos datos servirán para las comparaciones de las colectas futuras.Las especies arbóreas del sistema silvopastoril, que se encuentran en desarrollo, se distribuyen en franjas siguiendo las curvas de nivel (Sánchez Matta et al., 2009) y con un ancho de 10 metros (Ver Figura 1).

La contabilización de la biota se realizó entre las franjas, en cuadrantes de $30 \mathrm{~cm}$ de largo, $30 \mathrm{~cm}$ de ancho y $30 \mathrm{~cm}$ de profundidad, con un total de 30 muestras por cada población muestral. La biota fue colectada y luego identificada en laboratorio a nivel de órdenes, con el apoyo de especialistas y de bibliografía especializada. En cada población muestral se realizó: cálculo de abundancia relativa, índice de riqueza de Margalef, índice de dominancia de Berger-Parker, índice de dominancia de Simpson e índice de biodiversidad de Shannon, seleccionados por ser cuantificables y útiles para el análisis de diversidad alfa de las especies (Chiquin Baños y Velecela Caiza, 2015).

\section{Índice de abundancia relativa}

$$
A R=\frac{\text { Número de individuos de una especie }}{\text { Total de individuos de todas las especies }} \times 100
$$




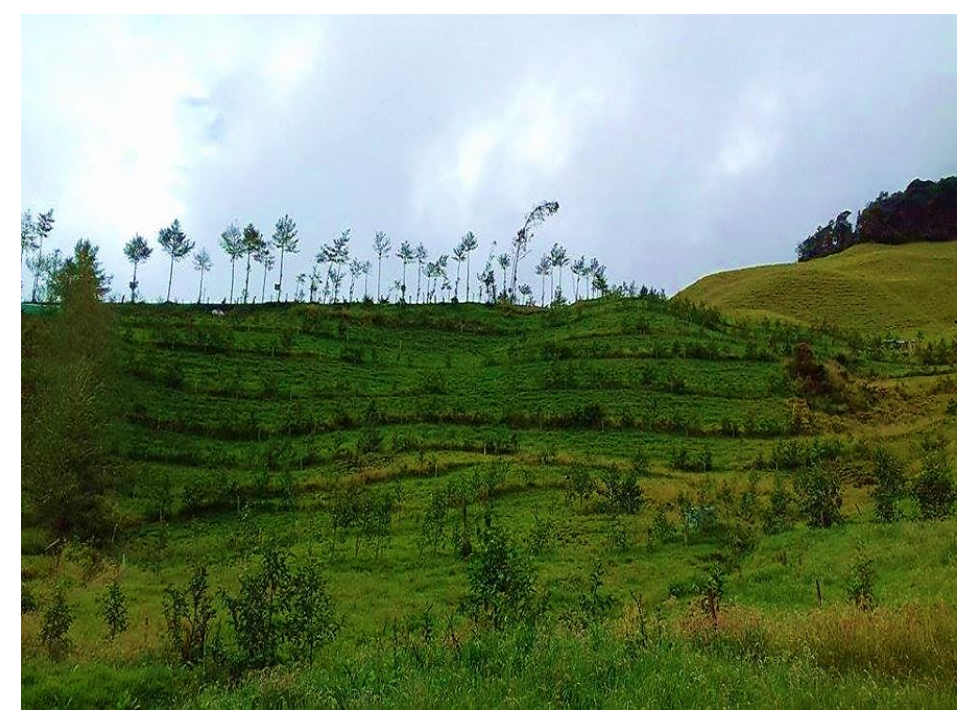

Figura 1. Distribución de las especies arbóreas en franjas en cada población muestral

En donde:

$\mathrm{AR}=$ Abundancia relativa

\section{Índice de riqueza de Margalef}

En donde:

$$
D=\frac{S-1}{\ln N}
$$

$\mathrm{D}=$ Índice de Margalef

$\mathrm{S}=$ Número de especies

ln = logaritmo natural

$\mathrm{N}=$ Número total de individuos

\section{Índice de Berge - Parker}

$$
B=\frac{N_{m a ́ x}}{N}
$$

En donde:

$\mathrm{B}=$ Índice de Berger - Parker

Nmáx = Número de individuos del taxón más abundante.

$\mathrm{N}=$ Número total de individuos de la muestra.

\section{Índice de diversidad de Simpson}

$$
D_{s}=\frac{n(n-1)}{N(N-1)}
$$

En donde:
Ds $=$ Diversidad de Simpson .

$\mathrm{n}=$ Número de individuos de la especie.

$\mathrm{N}=$ Total de individuos.

Debido a que Ds y la diversidad están negativamente relacionadas, el índice de Simpson se expone como el recíproco o complemento (1-D), de tal forma que, si el índice aumenta, la diversidad también aumenta.

\section{Índice de diversidad de Shannon - Wiener}

$$
H=-\sum_{i=1}^{s} p_{i} \times \ln p_{i}
$$

En donde:

$\mathrm{H}=$ Índice de Shannon.

$\mathrm{pi}=$ proporción de especies.

$\mathrm{S}=$ número de especies (riqueza de especies).

$\ln =$ Logaritmo natural.

Tales índices sirven para calcular la diversidad alfa, aplicables a zonas con riqueza de especies consideradas homogéneas (Moreno, 2001), en la zona de estudio con características similares de altitud, climáticas y edáficas. 


\section{Resultados y discusión}

\section{Índice de abundancia relativa}

Corresponde al número total de individuos de una especie expresada como una proporción del número total de individuos de todas las especies (Moreno, 2001).

De acuerdo a los datos presentados en las Tablas 1,2 y 3 se identifica en las tres poblaciones muestrales, que el taxón de mayor proporción es Haplotaxida (lombrices de tierra), su presencia en el nivel trófico de consumidor primario aporta al rompimiento del material vegetal e incorporación de nutrientes, además de influir en los procesos de aireación y remoción de suelo (Adi-Saab Arrieche, 2012).

Es relevante el taxón Haplotaxida, para evaluar las perturbaciones ambientales y las medidas a tomar en cuenta en dicho suelo. Identificar cambios en la diversidad, número, distribución de las especies o la dominancia, alertan acerca de procesos empobrecedores Magurran (2004), que no es el caso de la zona de estudio, ya que los resultados analizados muestran números adecuados en el taxón Haplotaxida.

Tabla 1. Abundancia relativa de la población muestral de Aliso (Alnus acuminata) y mezcla forrajera

\begin{tabular}{clccc}
\hline $\mathbf{N}^{\circ}$ & Orden Taxonómico & Nombre común & Cantidad & $\begin{array}{c}\text { Abundancia } \\
\text { Relativa (\%) }\end{array}$ \\
\hline 1 & Haplotaxida & Lombrices de tierra & 396 & 76,89 \\
2 & Pulmonata & Babosas & 48 & 9,32 \\
3 & Geophilomorpha & Ciempiés & 1 & 0,19 \\
4 & Araneae & Arañas & 21 & 4,08 \\
5 & Coleopteros & Escarabajos & 18 & 3,5 \\
6 & Dermaptera & Tijeretas & 1 & 0,19 \\
7 & Diptera & Moscas, sancudos & 23 & 4,47 \\
8 & Lepidoptera & Mariposas y orugas & 3 & 0,58 \\
9 & Orthoptera & Saltamontes & 4 & 0,78 \\
& & TOTAL & 515 & 100 \\
\hline
\end{tabular}

Tabla 2. Abundancia relativa de la población muestral de Acacia (Acacia melanoxilon) y mezcla forrajera

\begin{tabular}{clccc}
\hline $\mathbf{N}^{\circ}$ & Orden Taxonómico & Nombre común & Cantidad & $\begin{array}{c}\text { Abundancia } \\
\text { Relativa (\%) }\end{array}$ \\
\hline 1 & Haplotaxida & Lombrices de tierra & 372 & 66,55 \\
2 & Pulmonata & Babosas & 17 & 3,04 \\
3 & Geophilomorpha & Ciempiés & 2 & 0,36 \\
4 & Araneae & Arañas & 47 & 8,41 \\
5 & Coleopteros & Escarabajos & 61 & 10,91 \\
6 & Diptera & Moscas, sancudos & 48 & 8,59 \\
7 & Lepidoptera & Mariposas y orugas & 2 & 0,36 \\
8 & Orthoptera & Saltamontes & 7 & 1,25 \\
9 & Hemipteros & Chinches y pulgones & 3 & 0,54 \\
& & TOTAL & 559 & 100 \\
\hline
\end{tabular}

La Granja: Revista de Ciencias de la Vida 27(1) 2018:77-85. 
Tabla 3. Abundancia relativa de la población muestral de Testigo mezcla forrajera

\begin{tabular}{cllcc}
\hline $\mathbf{N}^{\circ}$ & Orden Taxonómico & Nombre común & Cantidad & $\begin{array}{c}\text { Abundancia } \\
\text { Relativa (\%) }\end{array}$ \\
\hline 1 & Haplotaxida & Lombrices de tierra & 449 & 77,15 \\
2 & Pulmonata & Babosas & 15 & 2,58 \\
3 & Isopoda & Cochinillas & 1 & 0,17 \\
4 & Araneae & Arañas & 23 & 3,95 \\
5 & Coleopteros & Escarabajos & 45 & 7,73 \\
6 & Diptera & Moscas, sancudos & 34 & 5,84 \\
7 & Lepidoptera & Mariposas y orugas & 3 & 0,52 \\
8 & Orthoptera & Saltamontes & 7 & 1,2 \\
9 & Hemipteros & Chinches y pulgones & 3 & 0,52 \\
10 & Hymenoptera & Abejas y avispas & 2 & 0,34 \\
& & TOTAL & 582 & 100 \\
\hline
\end{tabular}

\section{Índice de riqueza de Margalef}

Se obtiene al conocer el número total de especies, en relación a total de los individuos de las especies presentes dentro de la muestra (Moreno, 2001), (ver Tabla 4).

Tabla 4. Índice de riqueza de Margalef

\begin{tabular}{cc}
\hline Población muestral & Índice \\
\hline Aliso (Alnus acuminata) y mezcla forrajera & 1,2812 \\
Acacia (Acacia melanoxilon) y mezcla forrajera & 1,2645 \\
Mezcla forrajera & 1,4136 \\
\hline
\end{tabular}

El mínimo valor que puede adoptar es cero, y ocurre cuando solo existe una especie en la muestra $(s=1$, por lo que $s-1=0)$. Valores bajos hacen referencia a ecosistemas con poca biodiversidad en relación a valores altos que son sitios con mayor presencia de especies.

Cabe anotar que los sitios de muestreo tienen el fin de pastoreo para producción de leche, por lo que es considerado como pecuario. Se puede observar la menor riqueza de especies en la población muestral acacia-pasto.

\section{Índice de Berger - Parker}

Este índice mide la dominancia de la especie más abundante, tal como menciona (Moreno, 2001), (ver Tabla 5).
Tabla 5. Índice de riqueza de Berger - Parker

\begin{tabular}{cc}
\hline Población muestral & Índice \\
\hline Aliso (Alnus acuminata) y mezcla forrajera & 0.769 \\
Acacia (Acacia melanoxilon) y mezcla forrajera & 0.665 \\
Mezcla forrajera & 0.771 \\
\hline
\end{tabular}

Este índice adquiere valores comprendidos entre 0 y 1 ( $0 \%$ y $100 \%)$. En la población muestral, el mayor índice se localiza en el testigo, es decir que existe más equidad en las poblaciones, pero con una disminución de la dominancia Moreno (2001).

\section{Índice de diversidad de Simpson}

Según Franco López (1985), este índice permite estimar la probabilidad de que dos individuos elegidos al azar en una comunidad provengan de diferentes especies, (ver Tabla 6).

Tabla 6. Índice de diversidad de Simpson

\begin{tabular}{cc}
\hline Población muestral & Índice \\
\hline Aliso (Alnus acuminata) y mezcla forrajera & 0,396 \\
Acacia (Acacia melanoxilon) y mezcla forrajera & 0,531 \\
Mezcla forrajera & 0,394 \\
\hline
\end{tabular}

De acuerdo al resultado del índice de Simpson se deduce que en la población muestral de acacia con mezcla forrajera se podrían encontrar, con mayor probabilidad, a los individuos de la misma especie en extracciones distintas.

\section{Índice de diversidad de Shannon - Wiener}


De acuerdo a Moreno (2001), este índice asume que los individuos son seleccionados al azar y que todas las especies están representadas en la muestra , (ver Tabla 7).

Tabla 7. Índice de diversidad de Shannon - Wiener

\begin{tabular}{cc}
\hline Población muestral & Índice \\
\hline Aliso (Alnus acuminata) y mezcla forrajera & 0,902 \\
Acacia (Acacia melanoxilon) y mezcla forrajera & 1,161 \\
Mezcla forrajera & 0,924 \\
\hline
\end{tabular}

Como indica Moreno (2001), este índice puede variar entre cero (cuando existe una sola especie) y el logaritmo de $S$ (cuando las especies están representadas por el mismo número de individuos), identificando de esta manera en los resultados del índice de Shannon-Wiener que la población muestral con mayor valor de diversidad se encuentra en la parcela de acacia con la mezcla forrajera.

Hay que considerar que la población muestral tiene el objetivo de pastoreo y las especies vegetales dominantes son los pastos, lo que influye en la poca presencia de especies de fauna edáfica, de ahí que los valores en conjunto denotan bajos índices en las tres poblaciones muestrales. El resultado del índice de Shannon se puede contrastar con aquellos ecosistemas muy biodiversos los mismos que pueden llegar a tener valores cercanos a cinco.

Dado que las condiciones climáticas influyen en el desarrollo de las plantas, que a su vez determinan el crecimiento de la fauna, los cambios en el número de especies pueden variar de una época a otra respecto a temperatura y precipitación. Según menciona Lazo et al. (2007) el número de individuos de artrópodos es diferente en periodos de distinta precipitación, es necesario precisar entonces que para el caso de la finca "San Vicente", la contabilización se la realizó en los meses de menor precipitación y los resultados del conteo son de una primera etapa, a futuro se realizarán mediciones que permitan comparar el número de especies con meses de mayor precipitación en distintas épocas del año.

El análisis de índices bióticos en la finca "San Vicente" se basó en la diversidad alfa, ya que el sitio de estudio se lo considera homogéneo porque presenta las mismas condiciones edáficas, climáticas, altitud, y con sistemas silvopastoriles, en concordancia con lo expuesto por Whitaker (1972) la diversidad alfa es una comunidad homogénea, la diversidad beta es un grado de cambio entre diferentes comunidades en un paisaje, y la diversidad gamma es la riqueza de especies del conjunto de comunidades que integran un paisaje, resultante de las diversidades alfa y beta.

Debido a que los números poblacionales son variables en cada unidad geográfica, en cada paisaje, los análisis de biodiversidad son útiles para comprender la evolución del crecimiento de especies en función de perturbaciones ambientales o la influencia de las actividades humanas sobre ellas, es así que se usó los índices de biodiversidad para analizar si hay cambios significativos entre poblaciones muestrales, pudiendo comprobar que no hay cambios significantes entre ellos, aun cuando las especies arbóreas están en crecimiento.

La zona de estudio posee pastizales con árboles que están en desarrollo, además, por las condiciones climáticas y de cobertura vegetal la mayoría de la biota contabilizada se encuentra en sombra en el sistema radicular de los pastos, por su parte Vega (2014) manifiesta que la mayor presencia de oligoquetos e insectos se encuentra en suelos con pastizales, cuyo mayor porcentaje pertenecen a los oligoquetos, aun tomando en cuenta las diferencias de altitud que existen entre las dos zonas de estudio.

Desde otro enfoque Hernández Chávez, Sánchez Cárdenas y Simón Guelmes (2008) señalan que la presencia de árboles crea un microclima acorde a las exigencias de los organismos. En tal sentido en la zona de análisis la presencia de la cobertura vegetal con mezcla forrajera en la zona de estudio, se convierte en el hábitat propicio para la biota del suelo, y la presencia de los alisos y acacias favorece la formación de microclimas para la fauna edáfica, así se puede observar en los datos la presencia de fauna edáfica, y que los valores varían escasamente entre poblaciones muestrales, teniendo mayor presencia de biota en el sistema de alisos con mezcla forrajera, le sigue en número el testigo, y con menor número el población muestral de acacia con mezcla forrajera, haciendo notar que existe retención de humedad distinta en las poblaciones muestrales por la topografía que puede incidir en la mayor o menor presencia de biota edáfica, tal como muestra la Figura 2.

Además, la presencia de materia orgánica es importante para la productividad, y la biota del suelo es justamente quien la aporta (Argel, 2006), se mantienen las condiciones óptimas del hábitat de las especies que aportan con la descomposición de restos vegetales, y a su vez, aportan al mejoramiento simbiótico con otras especies, incluso bacterias. En este 


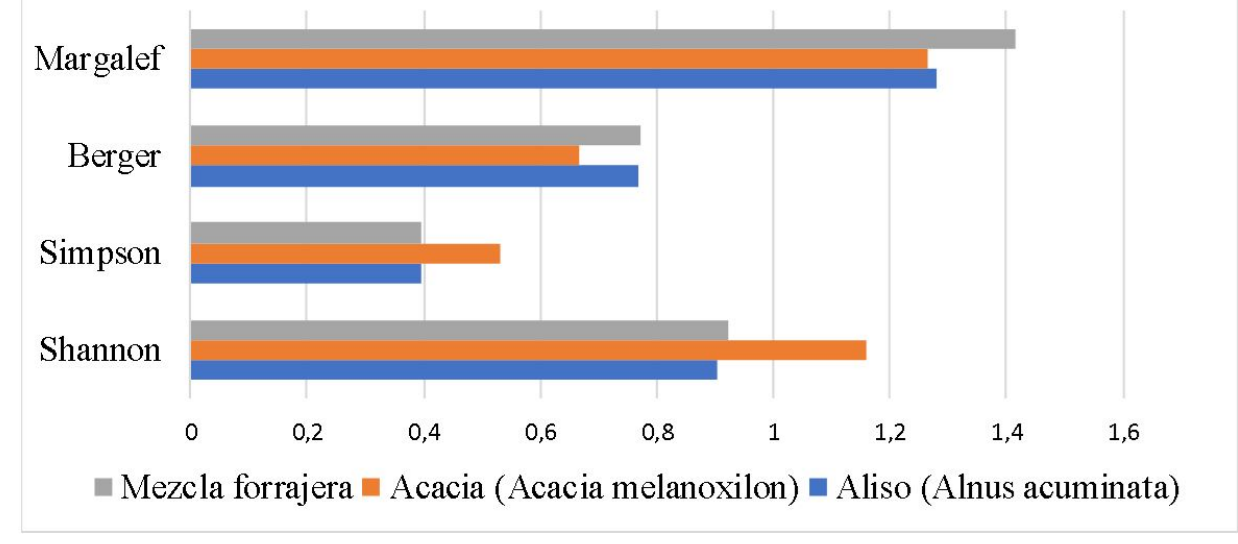

Figura 2. Índices bióticos en las muestras poblacionales

mismo tema, a criterio de Simón et al. (2005) el follaje de árboles leguminosos es una alternativa para el manejo de la fauna edáfica, la misma que es importante en los procesos de descomposición vegetal, degradación de celulosa, lignina y transformación en material húmico. Se deduce entonces que, a través del tiempo, la productividad del suelo de la zona de estudio se ha mantenido por la presencia de materia orgánica aportada por la biota edáfica.

En la zona de estudio el manejo del sistema silvopastoril garantiza las condiciones de vida de los organismos, su presencia es un indicador del ambiente óptimo del sistema, además, entre las tres poblaciones muestrales no existe una variación dilatada en el número de especies, lo que se puede deducir que la asociación de mezcla forrajera con árboles si aporta a las condiciones de vida de la fauna edáfica, los sistemas silvopastoriles propician la presencia de hábitats para insectos, se forman microclimas, y establecen interacciones equilibradas entre fitófagos, biorreguladores y microorganismos (Milera, 2013). En la investigación se realizó un análisis descriptivo propio de la investigación, sin recurrir a análisis estadísticos que midan la presencia de biodiversidad.

Finalmente, Cabrera (2012), reconoce como indicador de valoración del estado del suelo al comportamiento de poblaciones de la macrofauna edáfica, a través de su riqueza de especies. Se puede señalar entonces, que la fauna edáfica es un indicador de la calidad de los suelos y en la zona de estudio la presencia de la biota indica que los sistemas sil- vopastoriles están en buenas condiciones en las tres poblaciones muestrales, favoreciendo la productividad y las relaciones simbióticas entre las especies.

\section{Conclusiones}

Las aplicaciones de índices de biodiversidad permiten el análisis sobre el número de las poblaciones y sus especies, siendo herramientas útiles para observar los niveles de perturbación ambiental. Por otro lado, existe poca variabilidad en los índices de biodiversidad en las tres poblaciones muestrales, lo que denota uniformidad en las condiciones ambientales y edáficas, tomando en cuenta que las especies arbóreas aún están en crecimiento. Se identifica, además, que las condiciones físicas y químicas de las poblaciones muestrales se encuentran dentro de los parámetros deseados y la cantidad de fauna edáfica varía escasamente en espacio. De igual manera, la presencia de la fauna edáfica favorece los procesos de aireación, remoción de suelo e incorporación de nutrientes, mejorando la productividad de los suelos silvopastoriles. En consecuencia, es recomendable que la aplicación de los índices de biodiversidad se replique en otros sistemas silvopastoriles andinos, con el fin de realizar comparaciones futuras respecto a variaciones poblacionales. Así mismo, se debe mantener las condiciones actuales de los sistemas silvopastoriles para que se conserven las poblaciones de fauna de suelo, que permitan un monitoreo futuro sobre la evolución de comunidades edáficas. Finalmente, es necesario involucrar a 
docentes y estudiantes de niveles superiores en tareas de muestreo y contabilización de especies que aporten al análisis de condiciones ambientales y de la fauna edáfica.

\section{Referencias}

Adi-Saab Arrieche, R. 2012. Evaluación de la calidad del suelo, en el sistema productivo orgánico la Estancia, Madrid, Cundinamarca, 2012. Utilizando indicadores de calidad de suelos. In Tesis de grado. Pontificia Universidad Javeriana. [en línea] disponible en: https://goo.gl/3rX653.

Argel, P. J. 2006. “Contribución de los forrajes mejorados a la productividad ganadera en sistemas de doble propósito." Archivos Latinoamericanos de Producción Animal. 14(2):65-72. [en línea] disponible en: https://goo.gl/jBYmXo.

Cabrera, G. 2012. "La macrofauna edáfica como indicador biológico del estado de conservación/perturbación del suelo. Resultados obtenidos en Cuba." Pastos y Forrajes 35(4):346-363. [en línea] disponible en: https://goo.gl/wnKYkC.

Chiquin Baños, M. G y D. C. Velecela Caiza. 2015. Estudio de la diversidad florística y características del suelo dentro de un proceso de restauración activa luego de un período de lluvia, en la zona de influencia del "Cerro Puntas" nororiente de Quito. In Tesis de grado. Universidad Politécnica Salesiana. Ecuador. [en línea] disponible en: https://goo.gl/PbcoE2.

Franco López, J. 1985. Manual de Ecología. Cornell University. Trillas. [en línea] disponible en: https://goo.gl/kwLqb8.

Hernández Chávez, M, S Sánchez Cárdenas y L. Simón Guelmes. 2008. "Efecto de los sistemas silvopastoriles en la fertilidad edáfica." Zootecnia Tropical 26(3):319-321. [en línea] disponible en: https: //goo.gl/v14mFq.

Hernández Sampieri, R, C Fernández Collado y P. Baptista Lucio. 2014. Metodología de la Investigación. $6^{t a}$ ed. Mc Graw Hill Education. [en línea] disponible en: https://goo.gl/du8h5N.

Jordán López, A. 2005-2006. Manual de Edafología. Departamento de Cristalografía, Mineralogía y Química Agrícola de la Universidad de Sevilla. [en línea] disponible en: https:/ /goo.gl/1GyFk8.
Lazo, J. A, N. V Valdés, R. A Sampaio y G. L. D. Leite. 2007. "Diversidad zoológica asociada a un silvopastoreo leucaena-guinea con diferentes edades de establecimiento." Pesquisa Agropecuária Brasileira 42(12):1667-1674. [en línea] doi: http:/ / dx.doi.org/10.1590/S0100--204X2007001200001.

Magurran, A. 2004. Measuring Biological Diversity. Blackwell Science Ltd. [en línea] disponible en: https://goo.gl/3SBycP.

Milera, M. 2013. “Contribución de los sistemas silvopastoriles en la producción y el medio ambiente." Avances en Investigación Agropecuaria 17(3):7-24. [en línea] disponible en: https://goo. gl/KyjnPW.

Moreno, C. E. 2001. Métodos para medir la biodiversidad. M \& $\mathrm{T}-$ Manuales y Tesis SEA, vol. 1. CYTED, ORCYT - UNESCO, Sociedad Entomológica Aragoneza (SEA). [en línea] disponible en: https://goo.gl/phCgPk.

Sánchez Matta, L, G. M Amado Saavedra, P. J Criollo Campos, T Carvajal Salcedo, J Roa Triana, A Cuesta Peralta, A Conde Pulgarín, A Uma' na Arboleda, Luz M Bernal y L. Barreto de Escovar. 2009. El Aliso (Alnus acuminata H.B.K.) como alternativa silvopastoril en el manejo sostenible de praderas en el trópico alto colombiano. Corpoica. [en línea] disponible en: https://goo.gl/zAJ6Vj.

Simón, L, M Hernández, F Reyes y S. Sánchez. 2005. "Efecto de las leguminosas arbóreas en el suelo y en la productividad de los cultivos acompañantes." Pastos y Forrajes 28(1):29-45. Corpoica. [en línea] disponible en: https:/ /goo.gl/5eymYw.

Thevathasan, N, A Gordon, J Simpson, X Peng, S Silim, R Soolanayakanahally y H de Gooijer. 2014. "Sustainability Indicators of Biomass Production in Agroforestry Systems." The Open Agriculture Journal 8:1-11. Corpoica. [en línea] doi: http://dx. doi.org/10.2174/1874331501408010001.

Vega, A. 2014. "Evaluación de la macrofauna edáfica en un sistema silvopastoril en el Valle del Cauto." Revista Cubana de Ciencia Agrícola 48(2):189193. Corpoica. [en línea] disponible en: https:// goo.gl/5th1sU.

Whitaker, R. H. 1972. "Evolution and Measurement of Species Diversity." Taxon 21(2/3):213251. Corpoica. [en línea] doi: http://doi.org/10. $2307 / 1218190$. 